\title{
Solar Variability Manifestation on Earth-Climate Parameters Registered at a Tropical Location
}

\author{
Oscar Alvarez Pomares and Jorge Pérez Doval \\ Instituto de Geofísica y Astronomía, \\ Calle 212 No. 2906 e/ 29 y 31, La Coronela, \\ Ciudad de La Habana, CP 11600. \\ E-mail: oscaralv@geoastro.inf.cu, jpdoval@geoastro.inf.cu \\ Pedro A. Cárdenas Pérez and Raimundo Vega González \\ Centro Nacional del Clima, ISMET, Loma de Casablanca
}

\begin{abstract}
The authors present evidence for a connection between solar activity and mean winter temperatures at Havana, Cuba, in observations covering the past 130 years. The colder-than-average winters tend to follow $2 \pm 1$ years after years of maximum solar activity.
\end{abstract}

\section{Secular behavior}

A good agreement was observed between the behavior of the mean temperature (1871-1998) and mean atmospheric pressure (1909-1998) registered at Havana on one hand and the solar cycle length on the other. This outcome gives evidence that solar activity, or some agent closely related to it, have had a significant influence on the long term variation of the surface air mean temperature registered during more than a century, in a tropical location. This effect is more striking in winter- than in summertime. For the mean pressure the good correlation is noticeable for most of the past century.

A cause of this phenomenon can be the suggested relation between the cosmic rays arriving from the Sun to the Earth and cloud cover (Friis-Christensen \& Svensmark 1997). It is well known that the solar cycles of short duration (approximately 10 years) are generally very active, while the long cycles (longer than 11.5 years) appear to be less active. For that reason, one may infer that in the short cycles the decrease of cloud cover could be greater than during long cycles. Then the increase of temperature could, in consequence, be larger.

\section{Seasonal behavior}

We find that during the immediate years after the year of the maximum of the solar activity, a "cold winter" takes place, with a quarterly mean temperature of 0.9 to 1.4 degrees lower than any of the remaining years of the cycle. The effect is somewhat more significant for the highest mean temperatures. In the great majority of the cases, the minimum of temperature does take place $2 \pm 1$ year 
after the year of the solar maximum. All temperature minima take place in the first four years, and they appear not to be linked to temperature decreases caused by volcanic eruptions. During the months of full summer (July-September) no seasonal influence was observed.

The seasonal decrease of the temperature during winters, after the maxima of solar activity, can be associated to changes in the general atmospheric circulation of the North Hemisphere induced by the corpuscular radiation coming from the solar explosions which take place more frequently after the maximum of sunspots.

The temperature values in Cuba during the winters are ruled by the prevalence of the régime of meridian circulation, while in the summers the régime that prevails has a zonal character.

\section{Concluding remarks}

We take notice of how the secular behavior as well the seasonal one, shows a clearer manifestation related to the solar activity during the winter period, when the influence of the cold masses of air coming from the North plays a prevailing role in the climate of the region. On the other hand, in the summer period, or in rainy season, when the prevailing influence is from the quasi-stationary North Atlantic high pressure, and the tropical easterly waves arriving from the African western coast are a principal feature, the secular manifestations are seldom observed, and the seasonal ones have no significance. These results give evidence for the need to take in account the solar activity, to explain the climatic behavior in this region, both in long and medium terms.

\section{References}

Friis-Christensen, E. \& Svensmark, H. 1997, Adv. Space Res., 20, 913 